\title{
SPIN CORRELATION IN BINARY SYSTEMS
}

\author{
N. Farbiash ${ }^{1}$ and R. Steinitz ${ }^{1}$
}

\section{RESUMEN}

Examinamos la correlación entre las velocidades de rotación proyectadas en sistemas binarios. Este estudio es una extensión de un trabajo previo (Steinitz y Pyper, 1970; Levato, 1974). Una base de datos más amplia y nuevas pruebas nos permiten concluir que, en efecto, existe una correlación entre las velocidades de rotación proyectadas de las binarias. Sugerimos, de hecho, que los espines están correlacionados.

\begin{abstract}
We examine the correlation of projected rotational velocities in binary systems. It is an extension of previous work (Steinitz and Pyper, 1970; Levato, 1974). An enlarged data basis and new tests enable us to conclude that there is indeed correlation between the projected rotational velocities of components of binaries. In fact we suggest that spins are already correlated.
\end{abstract}

Key Words: BINARIES: GENERAL — STARS: ROTATION

\section{INTRODUCTION}

Evolution of binary systems could be a result of two main processes: three body collision, or evolving of binaries in one disk. The probability for three body collision is extremely small; thus it is more likely that binary systems evolve in one disk. In that case we expect to find correlation between the measured $V_{e} \sin (i)$ values of the members of such a system. Therefore, we study the degree of projected rotational velocity correlation between members of binary systems.

Slettebak (1963) did not find any significant difference between mean rotational velocity distribution of members of binary systems and those of single stars. Further, Abt (2001) concluded that spin axes are probably randomly oriented. On the other hand, Steinitz and Pyper (1970) concluded that some correlation of projected rotational velocities is present for the components of visual binaries. Levato (1974) also discussed this issue for visual binaries and close binary systems and found that there is indeed correlation of projected rotational velocities in binary systems. We now extend the original study (Steinitz and Pyper, 1970), which included only 50 systems. The significance of our results stems from the use of 1010 binary systems.

Actually, we will examine three samples of binary systems, as defined in the next section.

\section{DATA}

The Catalogue of Stellar Projected Rotational Velocities (Glebocki, Gnacinski \& Stawikowski 2000)

\footnotetext{
${ }^{1}$ Ben-Gurion University, Beer-Sheva, 84105, Israel.
}

is our source for the sample of binaries, chosen by imposing the following criteria:

1. The spectral type of both components is earlier than A9 (slow rotation of stars later than A9 would automatically simulate correlation).

2. Giants and supergiants may have lost their original rotational velocities. So we select only those binaries both of whose components are on the main sequence.

3. Multiple systems including more than two stars are excluded.

We are now left with a sample of 1010 real binary (RB) systems. Since the mean rotational velocity along the main sequence changes, it could happen that the choice of a sample restricted in spectral type will automatically exhibit the correlation we are looking for. To eliminate this possibility, and show that projected rotational velocity correlation is present in real binary systems only, we use two artificial samples of binaries. Define sample AB (Artificial Binaries) through shuffling the components of the real systems, and exclude the real ones (containing 2038180 systems). The second sample ABR (Artificial Binaries, Restricted), is obtained from sample $\mathrm{AB}$ by eliminating of all pairs having spectral type difference larger than two spectral subclasses (containing 263344 systems). This restriction is more stringent than the one we admit for the basic, real systems. 


\section{ILLUSTRATION}

In Fig.1. we plot the projected rotational velocities of one component against the other one, for all the three samples previously defined. From Fig.1. we see that samples $A B$ and $A B R$ do not exhibit any correlation whatsoever. In contrast, the correlation in sample RB is clearly evident. To quantify this result we apply more tests in the next section.

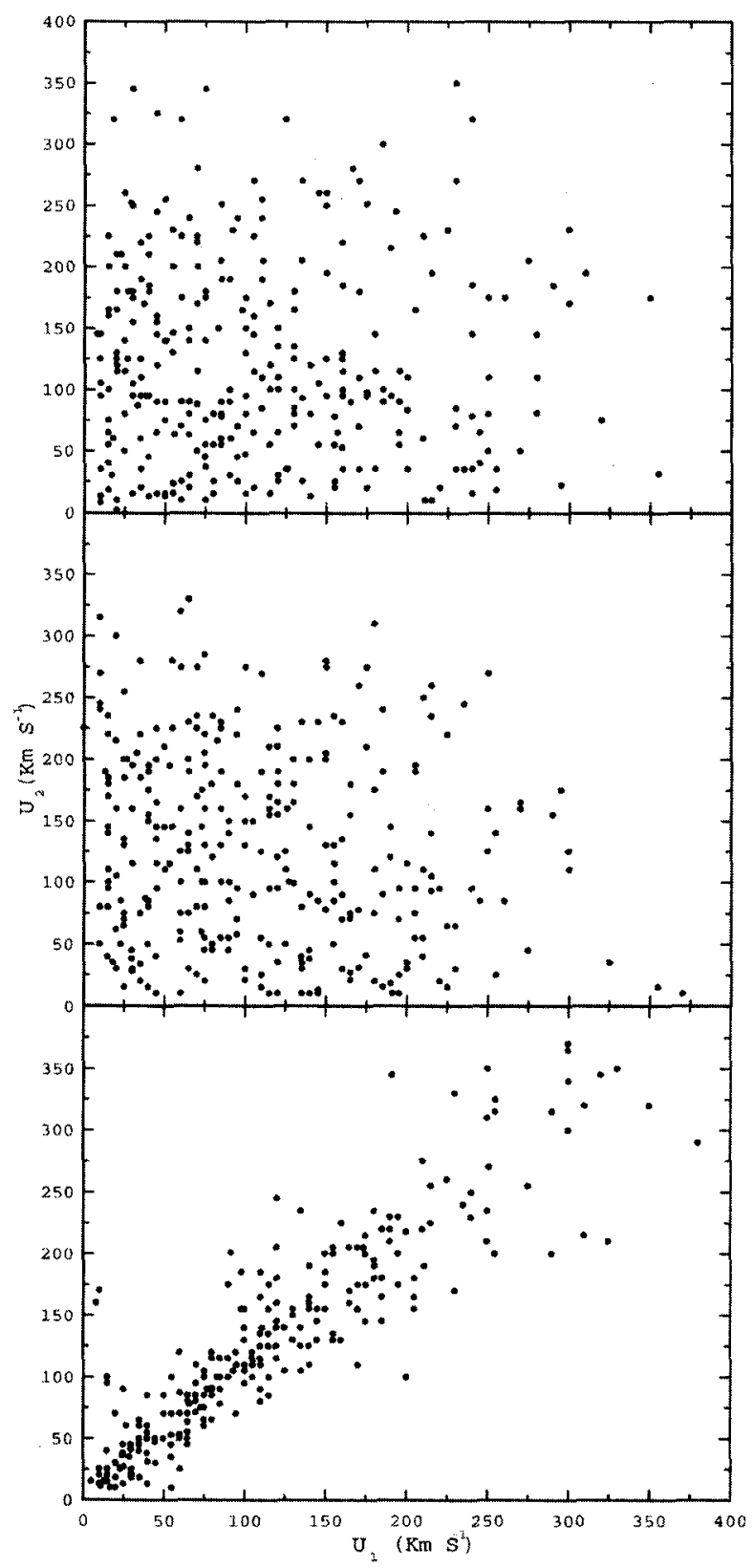

Fig. 1. Illustration of the projected rotational velocities of one component against the other for the samples $\mathrm{AB}$ (top), ABR (middle), and RB (bottom).

\section{ANALYSIS}

\subsection{Bivariate distribution, marginal distribution, and linear regression}

We prepare a table for each of the three samples, $\mathrm{AB}, \mathrm{ABR}$ and RB: it gives the discrete bivariate distribution of the samples. We give also the regression of the mean velocity of one component on the other one, as defined by equation (1).

$$
\bar{u}_{1}\left(u_{2}(j)\right)=\frac{\sum_{i=1}^{7} u_{1}(i) F\left(u_{1}(i), u_{2}(j)\right)}{\sum_{i=1}^{7} F\left(u_{1}(i), u_{2}(j)\right)} .
$$

Here $u_{1}=v_{1} \operatorname{sini}_{1}$ is the projected rotational velocity of the component, and $F\left(u_{1}, u_{2}\right)$ is the bivariate distribution.

For better illustration we show the regression of projected rotational velocity distribution of one component on the other one for each of the three samples. The $F\left(u_{1}, u_{2}\right)$ distributions for the samples $\mathrm{AB}, \mathrm{ABR}$, and $\mathrm{RB}$ are given in Table 1. The marginal distribution and the regression lines are also shown. The latter are plotted in Fig.2. For the ease of comparing the current results with previous ones, the velocity range has been divided into subintervals of $50 \mathrm{~km} \mathrm{~s}^{-1}$.

No significant differences between the main diagonal and other components of the table are evident for the samples $A B$ and $A B R$. But the set RB shows a distinct difference between the main diagonal and other elements of the table, indicating the presence of correlation of projected spins in real binaries.

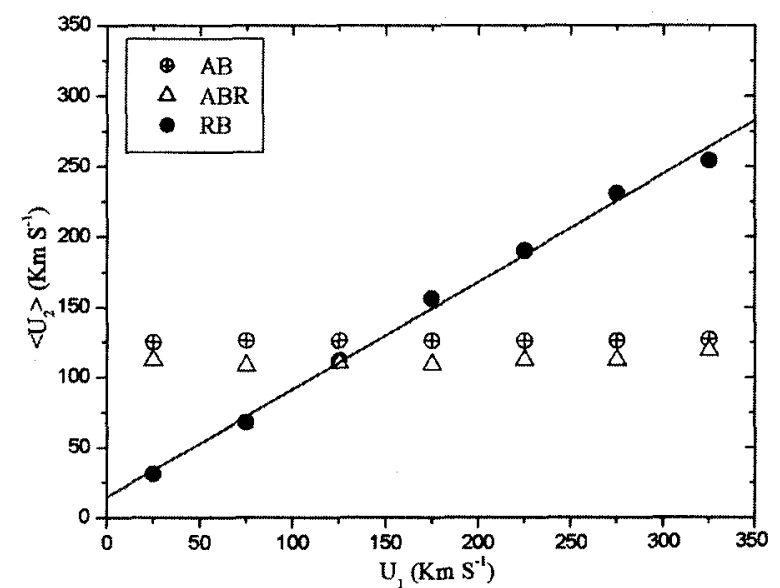

Fig. 2. Regression of the mean rotational velocities of one component (ordinate) as a function of the rotational velocity interval of the second component (abscissa) for samples AB (top), ABR (middle), and RB (bottom). 
TABLE 1

DISCRETE DISTRIBUTION FUNCTION $F\left(Y_{1}, Y_{2}\right)$ OF THE PROJECTED ROTATIONAL VELOCITIES OF THE COMPONENTS OF SAMPLE AB (TOP), ABR(MIDDLE), AND RB(BOTTOM).

\begin{tabular}{lccccccccc}
\hline \multicolumn{1}{c}{$u 1$} & $0-50$ & $50-100$ & $100-150$ & $150-200$ & $200-250$ & $250-300$ & $300-350$ & $\phi_{2}\left(u_{2}\right)$ & $\bar{u}_{1}\left(u_{2}\right)$ \\
\hline 02 & & & & & & & & & \\
\hline $0-50$ & 563 & 525 & 406 & 351 & 200 & 90 & 51 & 0.22 & 115 \\
$50-100$ & 617 & 575 & 446 & 388 & 221 & 99 & 58 & 0.24 & 116 \\
$100-150$ & 451 & 420 & 324 & 282 & 161 & 73 & 42 & 0.18 & 115 \\
$150-200$ & 433 & 404 & 313 & 271 & 155 & 70 & 41 & 0.17 & 116 \\
$200-250$ & 276 & 260 & 200 & 174 & 99 & 45 & 26 & 0.11 & 116 \\
$250-300$ & 122 & 115 & 88 & 77 & 44 & 20 & 12 & 0.05 & 116 \\
$300-350$ & 104 & 99 & 76 & 67 & 39 & 17 & 10 & 0.04 & 117 \\
$\phi_{1}\left(u_{1}\right)$ & 0.26 & 0.24 & 0.19 & 0.16 & 0.09 & 0.04 & 0.02 & & \\
$\bar{u}_{2}\left(u_{1}\right)$ & 125 & 126 & 126 & 126 & 126 & 126 & 127 & & \\
\hline $0-50$ & 576 & 496 & 385 & 356 & 205 & 105 & 0 & 0.21 & 112 \\
$50-100$ & 657 & 627 & 466 & 412 & 215 & 94 & 0 & 0.25 & 108 \\
$100-150$ & 452 & 447 & 331 & 277 & 141 & 56 & 22 & 0.17 & 110 \\
$150-200$ & 465 & 454 & 335 & 276 & 143 & 57 & 22 & 0.18 & 109 \\
$200-250$ & 290 & 264 & 201 & 172 & 96 & 45 & 18 & 0.11 & 112 \\
$250-300$ & 115 & 99 & 80 & 65 & 37 & 19 & 7 & 0.04 & 112 \\
$300-350$ & 115 & 88 & 74 & 66 & 43 & 25 & 10 & 0.04 & 119 \\
$\phi_{1}\left(u_{1}\right)$ & 0.27 & 0.25 & 0.19 & 0.16 & 0.09 & 0.04 & 0.01 & & \\
$\bar{u}_{2}\left(u_{1}\right)$ & 126 & 125 & 126 & 124 & 125 & 125 & 201 & & \\
\hline $0-50$ & 1901 & 198 & 20 & 0 & 0 & 0 & 0 & 0.21 & 31 \\
$50-100$ & 614 & 1564 & 158 & 40 & 10 & 0 & 0 & 0.24 & 68 \\
$100-150$ & 40 & 515 & 1000 & 158 & 0 & 0 & 0 & 0.17 & 112 \\
$150-200$ & 69 & 79 & 574 & 752 & 158 & 59 & 10 & 0.17 & 156 \\
$200-250$ & 10 & 50 & 99 & 535 & 347 & 50 & 40 & 0.11 & 190 \\
$250-300$ & 0 & 0 & 30 & 59 & 228 & 119 & 30 & 0.05 & 231 \\
$300-350$ & 0 & 10 & 10 & 50 & 129 & 198 & 89 & 0.05 & 254 \\
$\phi_{1}\left(u_{1}\right)$ & 0.26 & 0.24 & 0.19 & 0.16 & 0.09 & 0.04 & 0.02 & & \\
$\bar{u}_{2}\left(u_{1}\right)$ & 43 & 89 & 144 & 193 & 242 & 278 & 284 & & \\
\hline
\end{tabular}

${ }^{\mathrm{a}} u_{1}$ and $u_{2}$ are given in units of $\mathrm{km} \mathrm{s}^{-1}$.

\subsection{Modified convolution test}

Further illustration of the correlation between the rotational velocity distribution in real binary systems, and not in artificial ones, is to evaluate the modified convolution of the distribution functions. To validate the result, we first look at the single star velocity distribution, as shown in Fig. 3. The distribution is rather flat and does not indicate a strong maximum at any specific speed. Our modified convolution is essentially $g(u)=\int P(v-u \mid v) d v$, which in the discrete case is:

$$
g(u)=\sum_{v} F(v-u, v)
$$

As expected from the flatness of the distribution in Fig. 3., the convolution of artificial binary systems do not indicate a concentrated, sharp peak. However, for real binaries, there is a rather sharp peak present. It means that probability for the projected rotational velocity difference to be smaller than 50 $\mathrm{km} \mathrm{s}^{-1}$ is almost $60 \%$. The probability for the difference to be smaller than $100 \mathrm{~km} \mathrm{~s}^{-1}$ is more than $90 \%(!)$ in real binaries. 


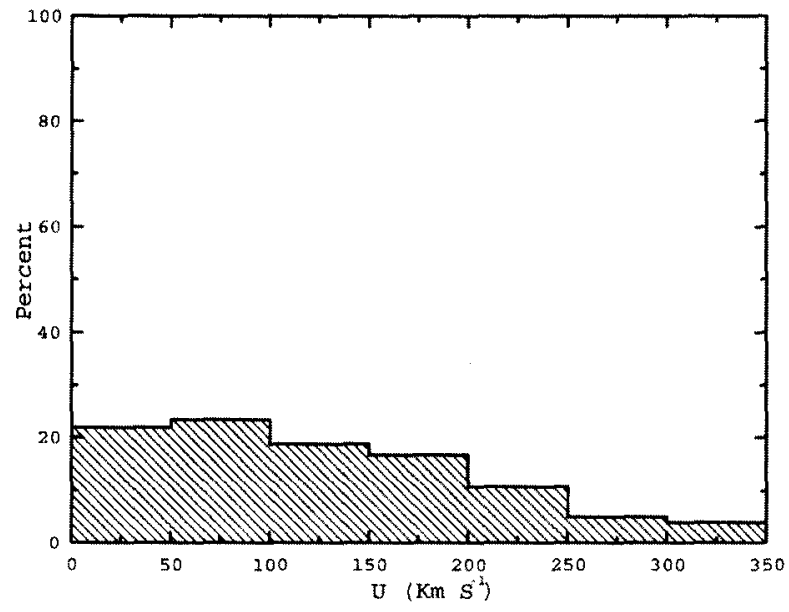

Fig. 3. Projected rotational velocity distribution of all components grouped into intervals of $50 \mathrm{~km} \mathrm{~s}^{-1}$.

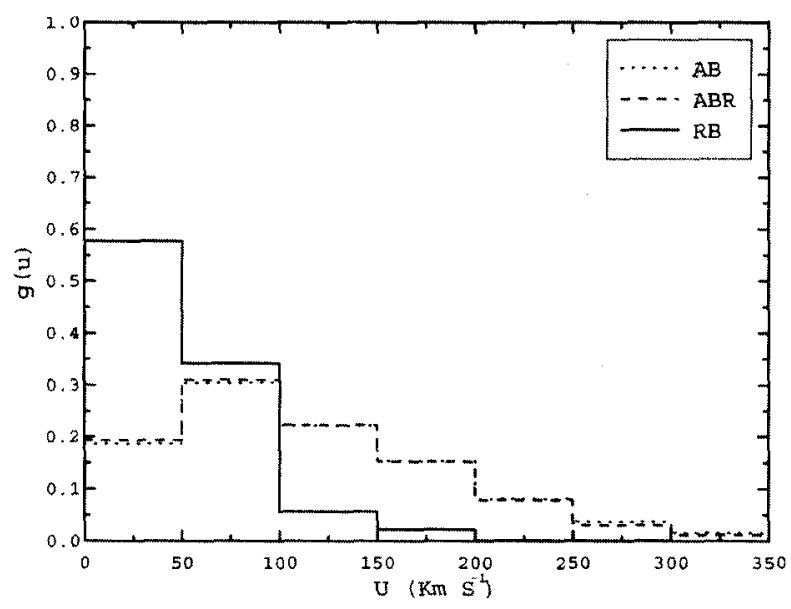

Fig. 4. Modified convolution of rotational velocities.

\section{CONCLUSIONS}

We examined three samples of binaries. For each of the samples we applied identical tests, to render significant comparisons between artificial binaries and real ones.

As expected, correlation in artificial systems (Set $A B$ and $A B R$ ) is insignificant. This is indicated by the minuscule slope of the regression lines. However, correlation for the binary set RB (Real Binaries) is clearly evident $\left(\mathrm{u}_{1}=.856 \mathrm{u}_{2}+32\right)$. One can describe our results roughly as follows:

$$
v_{1} \sin i_{1} \cong v_{2} \sin i_{2} \text {. }
$$

This relation can be understood either as:

1. $v_{1} \ll v_{2}$ while $\operatorname{sini} i_{1} \gg \operatorname{sini}_{2}$,

or

2. $v_{1} \simeq v_{2}$ as well as $\sin i_{1} \simeq \operatorname{sini} i_{2}$.

Since we have used a sample containing 1010 systems, the probability of the first case being real is extremely small. We rather accept the second explanation. We interpret this as meaning that:

1. Spin axes of members in binary systems are roughly parallel.

\section{Rotational speeds are correlated.}

There have been several investigations related to binary systems (Giuricin, Mardirossian \& Mezzetti, 1984a,b; Levato, 1976; Zahn, 1977; and Pan, 1997). These papers point out the importance of tidal interaction in close binary systems (especially the theory given by Zahn, 1966a,b,c, 1970, 1975, 1977).

We suppose that only a small fraction of the sample RB contains close binary systems. Thus we lack a general theory which can account for the empirical results demonstrated here.

\section{REFERENCES}

Abt, H. A. 2001, AJ, 122, 2008

Giuricin, G., Mardirossian, F., \& Mezzetti, M. 1984a, A\&A, 131, 152

Giuricin, G., Mardirossian, F., \& Mezzetti, M. 1984b, A\&A, 135, 393

Glebocki, R., Gnacinski, P., \& Stawikowski, A. 2000, AcA, 50, 509

Levato, H. 1974, A\&A, 35, 259

Levato, H. 1976, ApJ, 203, 680

Pan, K. 1997, A\&A, 321, 202

Slettebak, A. 1963, ApJ, 138, 118

Steinitz, R., \& Pyper, D. M., 1970, in Stellar Rotation, ed. A. Slettebak (IAU Coll. 4) (New York: Gordon \& Breach), 165

Zahn, J.-P. 1966a, AnAp, 29, 313

Zahn, J.-P. 1966b, AnAp, 29, 489

Zahn, J.-P. 1966c, AnAp, 29, 685

Zahn, J.-P. 1970, A\&A, 4, 452

Zahn, J.-P. 1975, A\&A, 41, 329

Zahn, J.-P. 1977, A\&A, 57, 383

N. Farbiash and R. Steinitz: Ben-Gurion University, Beer-Sheva, Israel 84105 (farbiash,steinitz@bgumail.bgu. ac.il). 


\section{DISCUSSION}

Sterzik - Could the spins be preferentially anti-parallel oriented to reach the same conclusion?

Farbiash - We have no practical way to say if spins are parallel or anti-parallel.

Mathieu - How does the correlation that you find depend on binary separation? Can you distinguish between axis alignment due to tidal effects as compared to other effects, such as formation?

Farbiash - We find very small differences between the correlation for spectroscopic binaries $(\sim 100$ systems $)$, visual ones ( $\sim 80$ systems), and other binaries we used in this study. The tidal effect is very important for close binaries, and is the main reason for synchronization among these systems. The reason we have made the Omega test is to see how many systems are already synchronized. We find that very few systems in the sample tested are synchronized. Data on the periods of the systems we tested are required in order to get a clear answer to the dependence between spin correlation and the binary separation.

Hanawa - Can you separate the correlation in $\sin i_{j}-\sin i_{k}$ and that in $V_{1}$ and $V_{2}$ ) by assuming, for example, that $\sin i$ has a random distribution?

Farbiash - The assumption that sin (i) is distributed randomly is probably true (Abt 2001). With the assumption that the spin axes are perfectly parallel (which is a very strong assumption!) we get a theoretical correlation value of $\approx 1$ for the rotation velocity. I have to be very careful saying that, since this value is meaningless if the spins are not perfectly parallel.

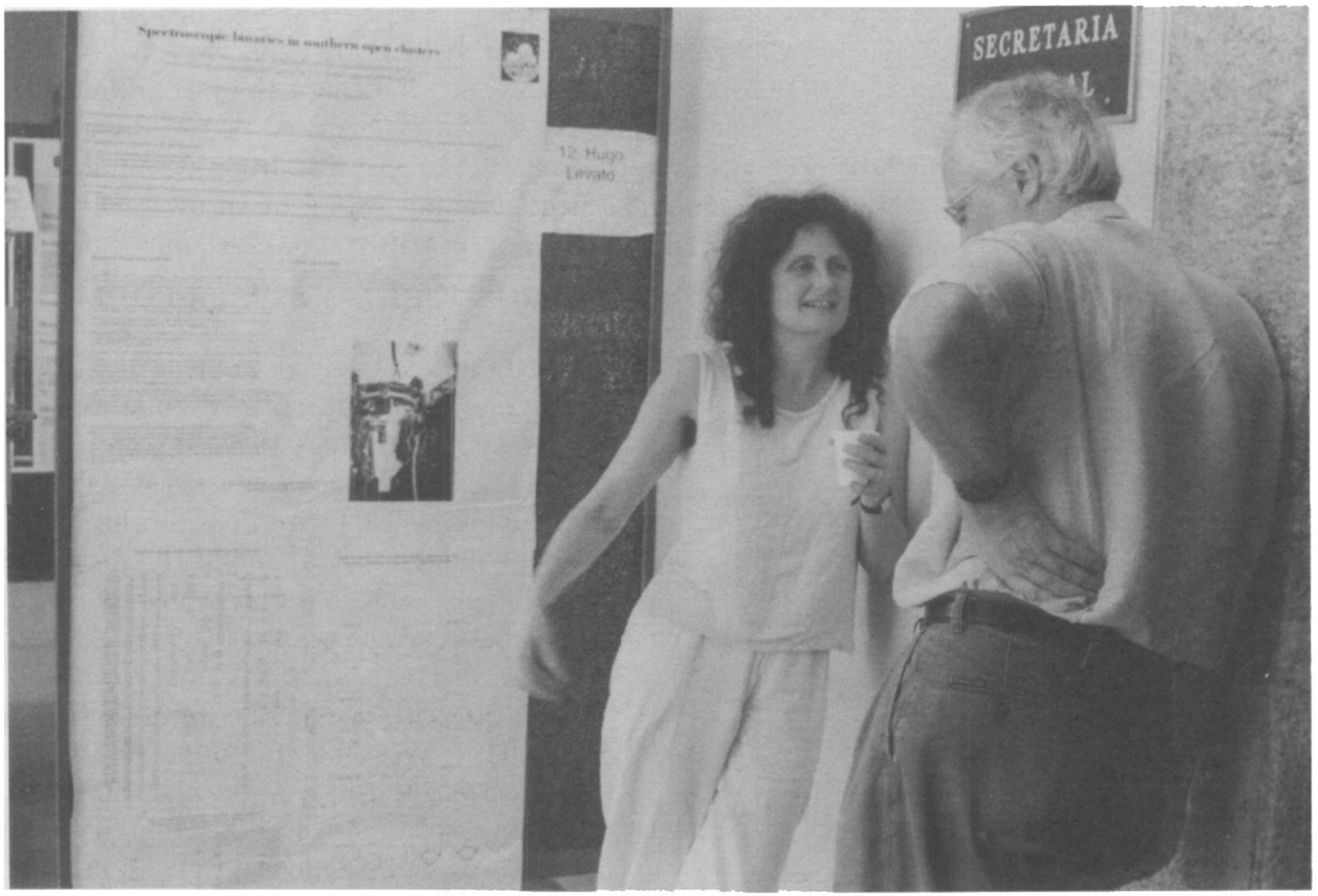

Cathie Clarke and Bo Reipurth. 